\title{
New Bounds on Poisson Approximation for Random Sums of Independent Binomial Random Variables
}

\author{
Giang Truong Le ${ }^{1}$ \\ ${ }^{1}$ University of Finance - Marketing, Vietnam \\ Correspondence: Giang Truong Le , University of Finance - Marketing, 2/4 Tran Xuan Soan street, District 7, Ho Chi \\ Minh city, Vietnam.
}

Received: April 10, 2018 Accepted: April 25, 2018 Online Published: June 19, 2018

doi:10.5539/ijsp.v7n4p43 URL: https://doi.org/10.5539/ijsp.v7n4p43

\begin{abstract}
In this paper, we use the Stein-Chen method to obtain new bounds on Poisson approximation for random sums of independent binomial random variables. Some results related to sums of independent binomial distributed random variables are also investigated. The received results in the present study are more general and sharper than some known results.
\end{abstract}

Keywords: Binomial random variable, Poisson approximation, Random sums, Stein-Chen method

\section{Introduction}

In recent times, Poisson approximation problem for random sums of discrete random variables has attracted the attention of mathematicians. Readers who are interested in this problem can refer to (Hung \& Giang, 2016b), (Kongudomthrap \& Chaidee, 2012), (Teerapabolarn, 2013), (Teerapabolarn, 2014b), (Vellaisamy \& Upadhye, 2009) and (Yannaros, 1991) for more details. We need to recall some results concerning the bounds in Poisson approximation for random sums of discrete random variables.

Let $Z_{1}, Z_{2}, \ldots$ be a sequence of independent Bernoulli random variables, each with probability of success $P\left(Z_{i}=1\right)=$ $p_{i}=1-P\left(Z_{i}=0\right), i=1,2, \ldots$, and let $N$ be a positive integer-valued random variable and independent of $Z_{i}$ 's. Let $U_{\lambda^{*}}$ be a Poisson random variable with mean $\lambda^{*}, V_{N}=\sum_{i=1}^{N} Z_{i}, \lambda^{*}=E\left(\lambda_{N}^{*}\right)$ and $\lambda_{N}^{*}=\sum_{i=1}^{N} p_{i}$. In 1991, Yannaros gave a uniform bound for the total variation distance between the distributions of $V_{N}$ and $U_{\lambda^{*}}$ as follows, see (Yannaros, 1991):

$$
d_{T V}\left(V_{N}, U_{\lambda^{*}}\right) \leq E\left|\lambda_{N}^{*}-\lambda^{*}\right|+E\left(\frac{1-e^{-\lambda_{N}^{*}}}{\lambda_{N}^{*}} \sum_{i=1}^{N} p_{i}^{2}\right) .
$$

Let $X_{1}, X_{2}, \ldots, X_{n}$ be $n$ independently distributed binomial random variables, each with probabilities

$$
P\left(X_{i}=k\right)=C_{r_{i}}^{k} p_{i}^{k}\left(1-p_{i}\right)^{r_{i}-k}
$$

where $p_{i} \in(0,1) ; r_{i}=1,2, \ldots ; i=1,2, \ldots n ; k=0,1, \ldots r_{i} ; C_{r_{i}}^{k}=\frac{r_{i} !}{k !\left(r_{i}-k\right) !}$.

Suppose that $N$ is a positive integer-valued random variable and independent of $X_{i}$ 's. Let $U_{\lambda}$ be a Poisson random variable with mean $\lambda, W_{N}=\sum_{i=1}^{N} X_{i}, \lambda_{N}=\sum_{i=1}^{N} r_{i} p_{i}$ and $\lambda=E\left(\lambda_{N}\right)$. In 2014, Teerapabolarn used the Stein-Chen method to obtain a uniform bound for the total variation distance between the distribution functions of $W_{N}$ and $U_{\lambda}$ as follows, see (Teerapabolarn, 2014a):

$$
d_{T V}\left(W_{N}, U_{\lambda}\right) \leq E\left(\frac{1-e^{-\lambda_{N}}}{\lambda_{N}} \sum_{i=1}^{N} r_{i} p_{i}^{2}\right)+\min \left\{1, \sqrt{\frac{2}{\lambda e}}\right\} E\left|\lambda_{N}-\lambda\right| .
$$

This paper is organized as follows. The second section is a brief introduction to Stein-Chen method. In section 3, we give main results of this paper, and conclusions of this study are presented in the last section.

In addition, throughout this paper, $d_{T V}$ is denoted the total variation distance, defined by

$$
d_{T V}(X, Y)=\sup _{A}|P(X \in A)-P(Y \in A)|,
$$

where $A \subseteq \mathbb{Z}_{+}:=\{0,1,2, \ldots\}$. 


\section{Preliminaries}

The Stein-Chen method has been dealt with in detail in many articles (the reader is referred to (Chen, 1975) and (Barbour, Holst \& Janson, 1992) for fuller development). The Stein-Chen method can be summarized as follows.

Let us denote by $F_{W_{n}}(A)$ the probability distribution function of a discrete random variable $W_{n} \in A$ and we will be denoted by $P_{\lambda_{n}}(A)=\sum_{k \in A} e^{-\lambda_{n}} \frac{\lambda_{n}{ }^{k}}{k !}$ the Poisson distribution function $\left(\lambda_{n}>0\right)$, defined on the set $A \subseteq \mathbb{Z}_{+}$. The best known method for estimating

$$
\Delta=\sup _{x}\left|F_{W_{n}}(A)-P_{\lambda_{n}}(A)\right|
$$

is basing on the following arguments (see (Chen, 1975) for more details).

Assume that $h$ is a bounded real-valued function defined on $\mathbb{Z}_{+}$and

$$
P_{\lambda_{n}}(h)=e^{-\lambda_{n}} \sum_{k=0}^{\infty} h(k) \frac{\lambda_{n}{ }^{k}}{k !} .
$$

Consider the function $f($.$) which is a solution of the Stein's equation$

$$
\lambda_{n} f(w+1)-w f(w)=h(w)-P_{\lambda_{n}}(h) .
$$

Setting

$$
h(w)=h_{A}(w)=\left\{\begin{array}{lll}
1, & \text { if } \quad w \in A, \\
0, & \text { if } \quad w \notin A .
\end{array}\right.
$$

Give $h=h_{A}$ and take the expectation of both sides of the equation (3), we have

$$
F_{W_{n}}(A)-P_{\lambda_{n}}(A)=E\left[\lambda_{n} f\left(W_{n}+1\right)-W_{n} f\left(W_{n}\right)\right] .
$$

Thus, the problem of estimating $\Delta$ can be reduced to that of estimating the difference of the expectations

$$
\left|E \lambda_{n} f\left(W_{n}+1\right)-E W_{n} f\left(W_{n}\right)\right| .
$$

According to Barbour et al. (see (Barbour, Holst \& Janson, 1992), for $C_{w-1}=\{0,1, \ldots, w-1\}$, the solution $f_{A}$ of (3) is of the form

$$
f_{A}(w)= \begin{cases}(w-1) ! \lambda_{n}{ }^{-w} e^{\lambda_{n}}\left[P_{\lambda_{n}}\left(h_{A \cap C_{w-1}}\right)-P_{\lambda_{n}}\left(h_{A}\right) P_{\lambda_{n}}\left(h_{C_{w-1}}\right)\right], & \text { if } w \geq 1, \\ 0, & \text { if } w=0 .\end{cases}
$$

Before starting the main results in next section, we also need the following lemmas, which is directly obtained from (Barbour, Holst \& Janson, 1992) and (Teerapabolarn \& Wongkasem, 2007).

Lemma 1 Let $V f_{A}(w)=f_{A}(w+1)-f_{A}(w)$. Then, for $A \subseteq \mathbb{Z}_{+}$and $k \in \mathbb{Z}_{+} \backslash\{0\}$,

$$
\sup _{w \geq k}\left|V f_{A}(w)\right| \leq \min \left\{\lambda_{n}^{-1}\left(1-e^{-\lambda_{n}}\right), \frac{1}{k}\right\} .
$$

Lemma 2 Let $w_{0} \in \mathbb{Z}_{+}$and $k \in \mathbb{Z}_{+} \backslash\{0\}$, we have

$$
\sup _{w \geq k}\left|V f_{C_{w_{0}}}(w)\right| \leq \lambda_{n}{ }^{-1}\left(e^{\lambda_{n}}-1\right) \min \left\{\frac{1}{w_{0}+1}, \frac{1}{k}\right\} .
$$

Lemma 3 Let $U_{\lambda_{N}}$ and $U_{\lambda}$ denote a Poisson random variable with mean $\lambda_{N}$ and $\lambda$, respectively. Then, for $A \subseteq \mathbb{Z}_{+}$, the total variation distance between the distributions of $U_{\lambda_{N}}$ and $U_{\lambda}$ satisfies the following inequality:

$$
d_{T V}\left(U_{\lambda_{N}}, U_{\lambda}\right) \leq \min \left\{1, \sqrt{\frac{2}{e \lambda}}\right\} E\left|\lambda_{N}-\lambda\right| .
$$

\section{Main Results}

The following lemma is established for proving the main results. 
Lemma 4 Let $X_{1}, X_{2}, \ldots$ be a sequence of independent binomial distributed random variables. Setting $W_{n}=\sum_{i=1}^{n} X_{i}$ and $\lambda_{n}=E\left(W_{n}\right)$. Then,

$$
\begin{aligned}
& E\left[\lambda_{n} f\left(W_{n}+1\right)-W_{n} f\left(W_{n}\right)\right] \\
& \quad=\sum_{i=1}^{n} \sum_{k \geq 1} k C_{r_{i}}^{k} p_{i}^{k+1}\left(1-p_{i}\right)^{r_{i}-k} E\left[f\left(W_{i}+k+1\right)-f\left(W_{i}+k\right)\right],
\end{aligned}
$$

where $f$ is a bounded real-valued function defined on $\mathbb{Z}_{+}$.

Proof. We have

$$
E\left[\lambda_{n} f\left(W_{n}+1\right)-W_{n} f\left(W_{n}\right)\right]=\sum_{i=1}^{n} E\left[r_{i} p_{i} f\left(W_{n}+1\right)-X_{i} f\left(W_{n}\right)\right] .
$$

Setting $W_{i}=W_{n}-X_{i}$,

$$
\begin{aligned}
& E\left[r_{i} p_{i} f\left(W_{i}+X_{i}+1\right)-X_{i} f\left(W_{i}+X_{i}\right)\right] \\
& =E\left[E\left[\left(r_{i} p_{i} f\left(W_{i}+X_{i}+1\right)-X_{i} f\left(W_{i}+X_{i}\right)\right) / X_{i}\right]\right] \\
& =E\left[r_{i} p_{i} f\left(W_{i}+1\right)\right] P\left(X_{i}=0\right) \\
& +E\left[r_{i} p_{i} f\left(W_{i}+2\right)-f\left(W_{i}+1\right)\right] P\left(X_{i}=1\right) \\
& +\sum_{k \geq 2} E\left[r_{i} p_{i} f\left(W_{i}+k+1\right)-k f\left(W_{i}+k\right)\right] P\left(X_{i}=k\right) \\
& =E\left[\left(r_{i} p_{i} P\left(X_{i}=0\right)-P\left(X_{i}=1\right)\right) f\left(W_{i}+1\right)\right] \\
& +\sum_{k \geq 2} E\left[\left(r_{i} p_{i} P\left(X_{i}=k-1\right)-k P\left(X_{i}=k\right)\right) f\left(W_{i}+k\right)\right] \\
& =E\left[\left(r_{i} p_{i}\left(1-p_{i}\right)^{r_{i}}-r_{i} p_{i}\left(1-p_{i}\right)^{r_{i}-1}\right) f\left(W_{i}+1\right)\right] \\
& +\sum_{k \geq 2} E\left[\left(r_{i} p_{i} C_{r_{i}}^{k-1} p_{i}^{k-1}\left(1-p_{i}\right)^{r_{i}-k+1}-k C_{r_{i}}^{k} p_{i}^{k}\left(1-p_{i}\right)^{r_{i}-k}\right) f\left(W_{i}+k\right)\right] \\
& =-E\left[r_{i} p_{i}^{2}\left(1-p_{i}\right)^{r_{i}-1} f\left(W_{i}+1\right)\right] \\
& +\sum_{k \geq 2} E\left[\left(r_{i} p_{i} C_{r_{i}}^{k-1} p_{i}^{k-1}\left(1-p_{i}\right)^{r_{i}-k+1}-\left(r_{i}-k+1\right) C_{r_{i}}^{k-1} p_{i}^{k}\left(1-p_{i}\right)^{r_{i}-k}\right) f\left(W_{i}+k\right)\right] \\
& =-E\left[r_{i} p_{i}^{2}\left(1-p_{i}\right)^{r_{i}-1} f\left(W_{i}+1\right)\right] \\
& +\sum_{k \geq 2} E\left[\left(\frac{r_{i}-k+1}{r_{i}} r_{i} p_{i} C_{r_{i}}^{k-1} p_{i}^{k-1}\left(1-p_{i}\right)^{r_{i}-k+1}-\left(r_{i}-k+1\right) C_{r_{i}}^{k-1} p_{i}^{k}\left(1-p_{i}\right)^{r_{i}-k}\right) f\left(W_{i}+k\right)\right] \\
& -\sum_{k \geq 2} E\left[\left(\frac{r_{i}-k+1}{r_{i}}-1\right) r_{i} p_{i} C_{r_{i}}^{k-1} p_{i}^{k-1}\left(1-p_{i}\right)^{r_{i}-k+1} f\left(W_{i}+k\right)\right] \\
& =-E\left[r_{i} p_{i}^{2}\left(1-p_{i}\right)^{r_{i}-1} f\left(W_{i}+1\right)\right] \\
& -\sum_{k \geq 2} E\left[\left(r_{i}-k+1\right) C_{r_{i}}^{k-1} p_{i}^{k+1}\left(1-p_{i}\right)^{r_{i}-k} f\left(W_{i}+k\right)\right] \\
& -\sum_{k \geq 2} E\left[\left(\frac{r_{i}-k}{r_{i}}-1\right) r_{i} C_{r_{i}}^{k} p_{i}^{k+1}\left(1-p_{i}\right)^{r_{i}-k} f\left(W_{i}+k+1\right)\right] \\
& +E\left[r_{i} p_{i}^{2}\left(1-p_{i}\right)^{r_{i}-1} f\left(W_{i}+2\right)\right] \\
& =r_{i} p_{i}^{2}\left(1-p_{i}\right)^{r_{i}-1} E\left[f\left(W_{i}+2\right)-f\left(W_{i}+1\right)\right] \\
& +\sum_{k \geq 2} k C_{r_{i}}^{k} p_{i}^{k+1}\left(1-p_{i}\right)^{r_{i}-k} E\left[f\left(W_{i}+k+1\right)-f\left(W_{i}+k\right)\right] \\
& =\sum_{k \geq 1} k C_{r_{i}}^{k} p_{i}^{k+1}\left(1-p_{i}\right)^{r_{i}-k} E\left[f\left(W_{i}+k+1\right)-f\left(W_{i}+k\right)\right] .
\end{aligned}
$$

This finishes the proof. 
The following theorems present non-uniform and uniform bounds for the distance between the distribution functions of $W_{N}$ and $U_{\lambda}$, which are the expected results.

\subsection{A Uniform Bound on Poisson Approximation for Random Sums of Independent Binomial Random Variables}

Theorem 1 For $A \subseteq \mathbb{Z}_{+}$, we have

$$
\begin{aligned}
d_{T V}\left(W_{N}, U_{\lambda}\right) \leq E & \left(\sum_{i=1}^{N} \min \left\{\lambda_{N}^{-1}\left(1-e^{-\lambda_{N}}\right) r_{i}, \frac{1-\left(1-p_{i}\right)^{r_{i}}}{p_{i}}\right\} p_{i}^{2}\right) \\
+ & \min \left\{1, \sqrt{\frac{2}{\lambda e}}\right\} E\left|\lambda_{N}-\lambda\right| .
\end{aligned}
$$

Proof. Let $f=f_{A}$ be defined as in (5) and applying (4), we have

$$
\left|P\left(W_{n} \in A\right)-\sum_{k \in A} \frac{\lambda_{n}^{k} e^{-\lambda_{n}}}{k !}\right|=\left|E\left[\lambda_{n} f\left(W_{n}+1\right)-W_{n} f\left(W_{n}\right)\right]\right| .
$$

Taking account of Lemma 4 and Lemma 1, it follows that

$$
\begin{aligned}
& \left|E\left[r_{i} p_{i} f\left(W_{n}+1\right)-X_{i} f\left(W_{n}\right)\right]\right| \\
& \leq \sum_{k \geq 1} k C_{r_{i}}^{k} p_{i}^{k+1}\left(1-p_{i}\right)^{r_{i}-k} E\left|f\left(W_{i}+k+1\right)-f\left(W_{i}+k\right)\right| \\
& \leq \sum_{k \geq 1} k C_{r_{i}}^{k} p_{i}^{k+1}\left(1-p_{i}\right)^{r_{i}-k} \sup _{w \geq k}|V f(w)| \\
& \leq \sum_{k \geq 1} k C_{r_{i}}^{k} p_{i}^{k+1}\left(1-p_{i}\right)^{r_{i}-k} \min \left\{\frac{1-e^{-\lambda_{n}}}{\lambda_{n}}, \frac{1}{k}\right\} \\
& =\min \left\{\frac{1-e^{-\lambda_{n}}}{\lambda_{n}} p_{i} \sum_{k \geq 1} k C_{r_{i}}^{k} p_{i}^{k}\left(1-p_{i}\right)^{r_{i}-k}, p_{i} \sum_{k \geq 1} C_{r_{i}}^{k} p_{i}^{k}\left(1-p_{i}\right)^{r_{i}-k}\right\} \\
& =\min \left\{\frac{1-e^{-\lambda_{n}}}{\lambda_{n}} p_{i} \sum_{k \geq 1} k P\left(X_{i}=k\right), p_{i}\left(\sum_{k \geq 0} P\left(X_{i}=k\right)-\left(1-p_{i}\right)^{r_{i}}\right)\right\} \\
& =\min \left\{\frac{1-e^{-\lambda_{n}}}{\lambda_{n}} p_{i} E\left(X_{i}\right), p_{i}\left(1-\left(1-p_{i}\right)^{r_{i}}\right)\right\} .
\end{aligned}
$$

Thus,

$$
\left|E\left[r_{i} p_{i} f\left(W_{n}+1\right)-X_{i} f\left(W_{n}\right)\right]\right| \leq \min \left\{\lambda_{n}^{-1}\left(1-e^{-\lambda_{n}}\right) r_{i}, \frac{1-\left(1-p_{i}\right)^{r_{i}}}{p_{i}}\right\} p_{i}^{2} .
$$

Combining (8) with (9), gives

$$
d_{T V}\left(W_{n}, U_{\lambda_{n}}\right) \leq \sum_{i=1}^{n} \min \left\{\lambda_{n}^{-1}\left(1-e^{-\lambda_{n}}\right) r_{i}, \frac{1-\left(1-p_{i}\right)^{r_{i}}}{p_{i}}\right\} p_{i}^{2} .
$$

From Lemma 3 and (10), it follows the fact that

$$
\begin{aligned}
d_{T V}( & \left.W_{N}, U_{\lambda}\right)=\sum_{n=1}^{\infty} P(N=n) d_{T V}\left(W_{n}, U_{\lambda}\right) \\
\leq & \sum_{n=1}^{\infty} P(N=n)\left[d_{T V}\left(W_{n}, U_{\lambda_{n}}\right)+d_{T V}\left(U_{\lambda_{n}}, U_{\lambda}\right)\right] \\
= & \sum_{n=1}^{\infty} P(N=n) d_{T V}\left(W_{n}, U_{\lambda_{n}}\right)+d_{T V}\left(U_{\lambda_{N}}, U_{\lambda}\right) \\
\leq & \sum_{n=1}^{\infty} P(N=n) \sum_{i=1}^{n} \min \left\{\lambda_{n}^{-1}\left(1-e^{-\lambda_{n}}\right) r_{i}, \frac{1-\left(1-p_{i}\right)^{r_{i}}}{p_{i}}\right\} p_{i}^{2} \\
& +\min \left\{1, \sqrt{\frac{2}{\lambda e}}\right\} E\left|\lambda_{N}-\lambda\right|
\end{aligned}
$$




$$
\begin{aligned}
\leq E & \left(\sum_{i=1}^{N} \min \left\{\lambda_{N}^{-1}\left(1-e^{-\lambda_{N}}\right) r_{i}, \frac{1-\left(1-p_{i}\right)^{r_{i}}}{p_{i}}\right\} p_{i}^{2}\right) \\
& +\min \left\{1, \sqrt{\frac{2}{\lambda e}}\right\} E\left|\lambda_{N}-\lambda\right| .
\end{aligned}
$$

This finishes the proof.

Remark 1 For $r_{1}=r_{2}=\ldots=r_{n}=1$, we have a uniform bound on Poisson approximation for the random sums of independent Bernoulli random variables:

$$
d_{T V}\left(V_{N}, U_{\lambda^{*}}\right) \leq E\left(\lambda_{N}^{*-1}\left(1-e^{-\lambda_{N}^{*}}\right) \sum_{i=1}^{N} p_{i}^{2}\right)+\min \left\{1, \sqrt{\frac{2}{\lambda^{*} e}}\right\} E\left|\lambda_{N}^{*}-\lambda^{*}\right| .
$$

Remark 2 Let us consider:

$$
\min \left\{1, \sqrt{\frac{2}{\lambda^{*} e}}\right\} \leq 1
$$

and

$$
\min \left\{\frac{1-e^{\lambda_{N}}}{\lambda_{N}} r_{i}, \frac{1-\left(1-p_{i}\right)^{r_{i}}}{p_{i}}\right\} p_{i}^{2} \leq \frac{1-e^{\lambda_{N}}}{\lambda_{N}} r_{i} p_{i}^{2}
$$

Thus, the bounds in (7) and (11) are sharper than the bounds in (2) and (1), respectively.

Corollary 1 For $N=n \in \mathbb{Z}_{+}$is fixed, then $\lambda=\lambda_{n}=\sum_{i=1}^{n} r_{i} p_{i}$ and

$$
d_{T V}\left(W_{n}, U_{\lambda_{n}}\right) \leq \sum_{i=1}^{n} \min \left\{\lambda_{n}^{-1}\left(1-e^{-\lambda_{n}}\right) r_{i}, \frac{1-\left(1-p_{i}\right)^{r_{i}}}{p_{i}}\right\} p_{i}^{2} .
$$

Remark 3 The result (12) is a uniform bound on Poisson approximation for sums of independent binomial random variables. This bound is sharper than those reported in (Teerapabolarn, 2014a).

3.2 A Non-uniform Bound on Poisson Approximation for Random Sums of Independent Binomial Random Variables

Theorem 2 For $w_{0} \in \mathbb{Z}_{+}$, we have

$$
\begin{gathered}
\left|P\left(W_{N} \leq w_{0}\right)-P\left(U_{\lambda} \leq w_{0}\right)\right| \leq \min \left\{\frac{2 \lambda}{w_{0}+1}, \min \left\{1, \sqrt{\frac{2}{e \lambda}}\right\} E\left|\lambda_{N}-\lambda\right|\right\} \\
+E\left(\sum_{i=1}^{N} \lambda_{N}{ }^{-1}\left(1-e^{-\lambda_{N}}\right) \min \left\{\frac{e^{\lambda_{N}} r_{i}}{\left(w_{0}+1\right)}, \frac{\left(1-\left(1-p_{i}\right)^{r_{i}}\right) e^{\lambda_{N}}}{p_{i}}\right\} p_{i}^{2}\right) .
\end{gathered}
$$

Proof. For $C_{w}=\{0, \ldots, w\}$ and $w_{0} \in \mathbb{Z}_{+}$, let $h_{w_{0}}: \mathbb{Z}_{+} \rightarrow \mathbb{R}$ such that

$$
h_{{w_{0}}_{0}}(w)= \begin{cases}1 & \text { if } w \leq w_{0}, \\ 0 & \text { if } w>w_{0} .\end{cases}
$$

According to Barbour et al. (see (Barbour, Holst \& Janson, 1992) on p.7), the solution $f_{C_{w_{0}}}(w)$ of (3) is expressed in the form of

$$
f_{{w_{0}}_{0}}(w)=\left\{\begin{array}{ccc}
(w-1) ! \lambda_{n}^{-w} e^{\lambda_{n}}\left[P_{\lambda_{n}}\left(h_{C_{w_{0}}}\right) P_{\lambda_{n}}\left(1-h_{C_{w-1}}\right)\right] & , \text { if } & w_{0}<w, \\
(w-1) ! \lambda_{n}^{-w} e^{\lambda_{n}}\left[P_{\lambda_{n}}\left(h_{C_{w-1}}\right) P_{\lambda_{n}}\left(1-h_{C_{w_{0}}}\right)\right] & , \text { if } & w_{0} \geq w, \\
0 & , \text { if } & w=0 .
\end{array}\right.
$$

Given $f=f_{C_{w_{0}}}$ and $h=h_{C_{w_{0}}}$, the Stein's equation

$$
h_{\mathrm{C}_{0}}(w)-\sum_{k \leq w_{0}} e^{-\lambda_{n}} \frac{\lambda_{n}^{k}}{k !}=\lambda_{n} f(w+1)-w f(w) .
$$


Taking expectations of both sides, and applying Lemma 2 and Lemma 4, we have

$$
\begin{aligned}
& \left|P\left(W_{n} \leq w_{0}\right)-P\left(U_{\lambda_{n}} \leq w_{0}\right)\right| \\
& \leq \sum_{i=1}^{n}\left(\sum_{k \geq 1} k C_{r}^{k} p_{i}^{k+1}\left(1-p_{i}\right)^{r_{i}-k} E\left|f\left(W_{i}+k+1\right)-f\left(W_{i}+k\right)\right|\right) \\
& \leq \sum_{i=1}^{n}\left(\sum_{k \geq 1} k C_{r}^{k} p_{i}^{k+1}\left(1-p_{i}\right)^{r_{i}-k} \lambda_{n}^{-1}\left(e^{\lambda_{n}}-1\right) \min \left\{\frac{1}{w_{0}+1}, \frac{1}{k}\right\}\right) \\
& =\sum_{i=1}^{n} \lambda_{n}^{-1}\left(e^{\lambda_{n}}-1\right) \min \left\{\frac{p_{i} \sum_{k \geq 1} k P\left(X_{i}=k\right)}{w_{0}+1}, p_{i} \sum_{k \geq 1} P\left(X_{i}=k\right)\right\} \\
& =\lambda_{n}^{-1}\left(e^{\lambda_{n}}-1\right) \sum_{i=1}^{n} \min \left\{\frac{r_{i}}{w_{0}+1}, \frac{1-\left(1-p_{i}\right)^{r_{i}}}{p_{i}}\right\} p_{i}^{2} .
\end{aligned}
$$

Thus,

$$
\left|P\left(W_{n} \leq w_{0}\right)-P\left(U_{\lambda_{n}} \leq w_{0}\right)\right| \leq \lambda_{n}^{-1}\left(e^{\lambda_{n}}-1\right) \sum_{i=1}^{n} \min \left\{\frac{r_{i}}{w_{0}+1}, \frac{1-\left(1-p_{i}\right)^{r_{i}}}{p_{i}}\right\} p_{i}^{2} .
$$

In addition, by using Lemma 3, Teerapabolarn showed that (see (Teerapabolarn, 2013) for more details):

$$
\left|P\left(U_{\lambda_{N}} \leq w_{0}\right)-P\left(U_{\lambda} \leq w_{0}\right)\right| \leq \min \left\{\frac{2 \lambda}{w_{0}+1}, \min \left\{1, \sqrt{\frac{2}{e \lambda}}\right\} E\left|\lambda_{N}-\lambda\right|\right\} .
$$

Combining (14) and (15) gives

$$
\begin{aligned}
& \left|P\left(W_{N} \leq w_{0}\right)-P\left(U_{\lambda} \leq w_{0}\right)\right| \\
& \leq \sum_{n=0}^{\infty} P(N=n)\left|P\left(W_{n} \leq w_{0}\right)-P\left(U_{\lambda} \leq w_{0}\right)\right| \\
& \leq \sum_{n=0}^{\infty} P(N=n)\left|P\left(W_{n} \leq w_{0}\right)-P\left(U_{\lambda_{n}} \leq w_{0}\right)\right| \\
& \quad+\left|P\left(U_{\lambda_{N}} \leq w_{0}\right)-P\left(U_{\lambda} \leq w_{0}\right)\right| \\
& \leq \sum_{n=0}^{\infty} P(N=n) \frac{1-e^{-\lambda_{n}}}{\lambda_{n}} \sum_{i=1}^{n} \min \left\{\frac{r_{i} e^{\lambda_{n}}}{w_{0}+1}, \frac{\left(1-\left(1-p_{i}\right)^{r_{i}}\right) e^{\lambda_{n}}}{p_{i}}\right\} p_{i}^{2} \\
& \quad+\min \left\{\frac{2 \lambda}{w_{0}+1}, \min \left\{1, \sqrt{\frac{2}{e \lambda}}\right\} E\left|\lambda_{N}-\lambda\right|\right\} \\
& \leq E\left(\frac{1-e^{-\lambda_{N}}}{\lambda_{N}} \sum_{i=1}^{N} \min \left\{\frac{r_{i} e^{\lambda_{N}}}{w_{0}+1}, \frac{\left(1-\left(1-p_{i}\right)^{r_{i}}\right) e^{\lambda_{N}}}{p_{i}}\right\} p_{i}^{2}\right) \\
& \quad+\min \left\{\frac{2 \lambda}{w_{0}+1}, \min \left\{1, \sqrt{\frac{2}{e \lambda}}\right\} E\left|\lambda_{N}-\lambda\right|\right\} .
\end{aligned}
$$

This finishes the proof.

Remark 4 For $r_{1}=r_{2}=\ldots=r_{n}=1$, we have a non-uniform bound on Poisson approximation for the random sums of independent Bernoulli random variables:

$$
\begin{aligned}
\left|P\left(V_{N} \leq w_{0}\right)-P\left(U_{\lambda^{*}} \leq w_{0}\right)\right| \leq \min & \left\{\frac{2 \lambda^{*}}{w_{0}+1}, \min \left\{1, \sqrt{\frac{2}{e \lambda^{*}}}\right\} E\left|\lambda_{N}^{*}-\lambda^{*}\right|\right\} \\
& +E\left(\frac{\left(e^{\lambda_{N}^{*}}-1\right)}{\left(w_{0}+1\right) \lambda_{N}^{*}} \sum_{i=1}^{N} p_{i}^{2}\right) .
\end{aligned}
$$


Corollary 2 For $N=n \in \mathbb{Z}_{+}$is fixed, then $\lambda=\lambda_{n}=\sum_{i=1}^{n} r_{i} p_{i}$ and

$$
\left|P\left(W_{n} \leq w_{0}\right)-P\left(U_{\lambda_{n}} \leq w_{0}\right)\right| \leq \lambda_{n}^{-1}\left(e^{\lambda_{n}}-1\right) \sum_{i=1}^{n} \min \left\{\frac{r_{i}}{w_{0}+1}, \frac{1-\left(1-p_{i}\right)^{r_{i}}}{p_{i}}\right\} p_{i}^{2} .
$$

Remark 5 The result (17) is a non-uniform bound on Poisson approximation for sums of independent binomial random variables.

\section{Conclusions}

We conclude this paper with the following comments. Bounds for the distance between the distribution function of random sums of independent binomial random variables and an appropriate Poisson distribution function were obtained. The received results in this paper are sharper than those reported in (Teerapabolarn, 2014a), (Teerapabolarn, 2014b), and (Yannaros, 1991). Moreover, non-uniform bounds on Poisson approximation for sums (and random sums) of independent binomial random variables are given. The results will be more interesting and valuable if we discuss Poisson approximation for random sums of dependent binomial random variables. We shall continue studying this matter in our future research.

\section{References}

Barbour, A. D., Holst, L., \& Janson, S. (1992). Poisson Approximation. Clarendon Press-Oxford.

Chen, L. H. Y. (1975). Poisson approximation for dependent trials. Ann. Probab., 3(3), 534-545.

Hung, L. T., \& Giang, T. L. (2014). On bounds in Poisson approximation for integer-valued independent random variables. Journal of Inequalities and Applications. https://doi.org/10.1186/1029-242X-2014-291

Hung, L. T., \& Giang, T. L. (2016a). On bounds in Poisson approximation for distributions of independent negativebinomial distributed random variables. SpringerPlus. https://doi.org/10.1186/s40064-016-1710-y

Hung, L. T., \& Giang, T. L. (2016b). On the bounds in Poisson approximation for independent geometric distributed random variables. Bulletin of the Irannian Mathematical Society, 42(5), 1087-1096.

Kongudomthrap, S., \& Chaidee, N. (2012). Bounds in Poisson approximation of random sums of Bernoulli random variables. Journal of Mathematics Research, (4), 29-35.

Teerapabolarn K., \& Wongkasem P. (2007). Poisson approximation for independent geometric random variables. Int. Math. Forum, 2, 3211-3218.

Teerapabolarn, K. (2013). Improved bounds on Poisson approximation for independent binomial random summands. International Journal of Pure and Applied Mathematics, 89(1), 29-33.

Teerapabolarn, K. (2014a). Poisson approximation for independent binomial random variables. International Journal of Pure and Applied Mathematics, 93(6), 775-777.

Teerapabolarn, K. (2014b). Poisson approximation for random sums of independent binomial random variables. Applied Mathematical Sciences, 8(173), 8643-8646.

Vellaisamy, P., \& Upadhye, S. (2009). Compound negative binomial approximations for sums of random variables. Probab. Math. Statist., (29), 205 - 226.

Yannaros, N. (1991). Poisson approximation for random sums of Bernoulli random variables. Statistics $\mathcal{E}$ Probability Letters, (11), 161-165.

\section{Copyrights}

Copyright for this article is retained by the author(s), with first publication rights granted to the journal.

This is an open-access article distributed under the terms and conditions of the Creative Commons Attribution license (http://creativecommons.org/licenses/by/4.0/). 